\title{
A Tool for Verification and Validation of Neural Network Based Adaptive Controllers for High Assurance Systems
}

\author{
Pramod Gupta ${ }^{\dagger}$ and Johann Schumann ${ }^{\ddagger}$ \\ ${ }^{\dagger}$ QSS Inc., ${ }^{\ddagger}$ RIACS / NASA Ames, \{pgupta|schumann\}@email. arc.nasa. gov
}

\begin{abstract}
High reliability of mission- and safety-critical software systems has been identified by NASA as a high-priority technology challenge. We present an approach for the performance analysis of a neural network (NN) in an advanced adaptive control system. This problem is important in the context of safety-critical applications that require certification, such as fight software in aircraft. We have developed a tool to measure the performance of the $N N$ during operation by calculating a confidence interval (error bar) around the NN's output. Our tool can be used during pre-deployment verification as well as monitoring the network performance during operation. The tool has been implemented in Simulink and simulation results on a $F-15$ aircraft are presented.
\end{abstract}

\section{Introduction}

The benefits of newral network based systems are clear-a system that can rapidly adapt to a changing environment can solve many of the complex problems we face today. These systems are attracting increasing attention in applications where autonomy is an important feature or where it is virtually impossible to analyze, in advance, all possible environmental conditions that may arise during the mission. Control applications which need to react to catastrophic changes (e.g., a broken control surface of an aircraft) can also benefit from a neural network based system. The application of neural networks within subdisciplines of engineering is increasing but new methods are required to ensure the reliable deployment and operation of these systems. In a safety-critical application, no neural network based system will be used unless verification and validation $(\mathrm{V} \& \mathrm{~V})$ can demonstrate its reliability in a cost effective manner. The adaptive nature of neural networks requires a significantly different approach to $\mathrm{V} \& \mathrm{~V}$ methods. In order to perform all required V\&V activities, the software development process (e.g., as prescribed by IEEE standards) need to be adapted accordingly (see e.g., [I]). Nevertheless, V\&V of neural network based control systems is very difficult, because the underlying theory of machine learning usually does not provide enough mathematically rigorous help for verification. Therefore, verification and validation must be augmented by specifically tailored validation and dynamic monitoring tools (see $[4,5]$ ).

In this abstract, we focus on a method and tool to analyze the performance of a neural network during $\mathrm{V} \& \mathrm{~V}$ and during operation (as a monitoring device). We will illustrate our tool with an adaptive flight control system (FCS), as developed within the IFCS (Intelligent Flight Control System) project at NASA Dryden. The FCS (Fig. 1) is a straight-forward dynamic inverse controller: the pilot stick commands are mixed with the current sensor readings (airspeed, angle of attack, and altitude) to form the desired behavior of the aircraft. The dynamic inverse model then calculates the required actuator (e.g., aileron) movements to bring the aircraft into the desired state. In nominal operation, the entire control is established that way. If, however, the aerodynamics of the aircraft changes (e.g., due to a broken surface), there is a deviation between desired and actual state. The neural network is trained during operation to produce a correction signal $U_{A D}$ to minimize this deviation. The controller and the network with a $\Sigma \Pi$ architecture are described in detail in [3].

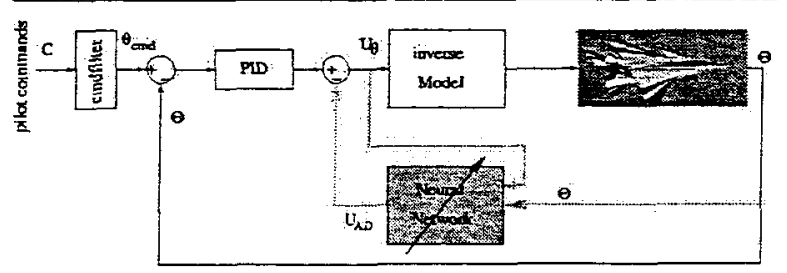

Figure 1. IFCS Adaptive Control Architecture 


\section{Confidence Measure and Confidence Tool}

For control applications, it is important that the controller's model of the plant reflects the actual system with sufficient accuracy - something which has to be demonstrated during $\mathrm{V} \& \mathrm{~V}$ of a neural networks based system. This means that it needs to be shown that for the entire operation envelope, the modeling error does not exceed a given threshold. Thus, to measure the reliability of the neural network output is of great interest. It is usually desirable that a statistical error bar is placed on the output of a neural network which predicts the probability how close the estimated and the actual values are. The error bar is a prediction of the range of the model output where the actual value lies with a probability of more than $95 \%$. With the assumption of a normal distribution of the errors, confidence intervals can be related to the standard deviation of the network output.

Using a Bayesian approach, the error bars can be calculated, given the history of training data and the parameters of the network (e.g., the weights). We have developed the tool CT/OLNN to dynamically calculate the error bars of the network outputs $U_{A D}$ for the controller shown in Fig. 1. It is implemented on top of the IFCS Simulink model for a F-15. The tool has been implemented to handle $\Sigma \Pi$ and single hidden layer neural networks. The tool can be modified easily to accommodate other network and control architectures for future applications.

\section{Simulation Results}

Figure 2 shows a graphical representation of the tool's output for three simulations runs for three different operating conditions. In each graph, the output of the NN over time is shown as a solid line. At time $t=1.5 \mathrm{~s}$, the pilot issues a command. In the nominal case (A), the neural network produces some small output to accommodate for modeling inaccuracies. During the time of adaptation, the confidence decreases but improves again over the time as neural network converges. In our figure, we show the error bars (variance) as dashed lines. Within this band, the actual (true) value is located with a probability of $95 \%$. A broad band corresponds to a low confidence value. Figures $2 \mathrm{~B}$ and $\mathrm{C}$ depict scenarios when a failure occurs (a control surface got stuck at different angles at $t=1.5 \mathrm{~s}$ ). In these cases, the NN's output needs to be substantially larger to counteract the damage. Also, the width of the error band is much larger, indicating a low confidence in the results of the network. The damage in Fig-

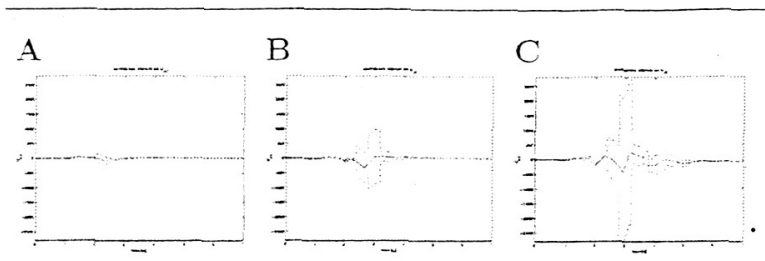

Figure 2. Output of the on-line adaptive ANN (solid); dashed lines define confidence interval

ure $2 \mathrm{C}$ was found to be already very close to the stability limit; the large waves in the output and the very low confidence is a clear indication of that.

\section{Conclusions}

The enormous potential of adaptive controllers for safety-critical applications is obvious; for example, potentially major catastrophic failures and loss of life can be avoided. Work on development of the NN-adaptive technology itself and on its V\&V methods is crucial for NASA missions as well as beneficial to numerous other agencies and the aerospace industry.

Our CT/OLNN analysis tool provides assistance to establish reliability of a neural network based adaptive control system. Our technique will enhance the understanding of performance of the adaptive systems and address the detection and possible prevention of catastrophic failures. However, for proper V\&V of such system, a multitude of technologies and tools are required. The development of such tools and processes will help to successfully infuse the neural network adaptive technology into real missions.

\section{References}

[1] D. Mackall, S. Nelson, and J. Schumann. Verification and Validation of Neural Networks of Aerospace Applications. Technical Report CR-211409, NASA, 2002.

[2] A. Mili, B. Cukic, Y. Liu, and R. Ayed. Towards the Verification and Validation of online adaptive Systems. In Comp. Methods in SW Eng. Kluwer, 2003.

[3] R. Rysdyk and A. Calise. Fault tolerant Flight Control via adaptive Neural Network augmentation. AIAA, AIAA98-4483:1722-1728, 1998.

[4] J. Schumann and P. Gupta. On V\&V of Neural Network based Controllers. In EANN 03, pp 40-47, 2003.

[5] J. Schumann and S. Nelson. Toward V\&V of Neural Network based Controllers. In WOSS, pp 67-72. ACM, 2002.

[6] S. Yerramalla, E. Fuller, M. Mladenovski, and B. Cukic. Lyapunov analysis of Neural Network Stability in an adaptive Flight Control System. In Proc. DSN'03, 2003. 